\title{
Sacubitril/Valsartan Improves Autonomic Function and Cardiopulmonary Parameters in Patients with Heart Failure with Reduced Ejection Fraction
}

\author{
Francesco Giallauria ${ }^{1, *(\mathbb{D})}$, Giuseppe Vitale $\left.{ }^{2} \mathbb{(}\right)$, Mario Pacileo $\left.{ }^{3} \mathbb{(}\right)$, Anna Di Lorenzo ${ }^{1}$, \\ Alessandro Oliviero ${ }^{1}$, Francesco Passaro ${ }^{1}$, Roberta Calce ${ }^{1}$, Alessandro Parlato ${ }^{1}$, \\ Crescenzo Testa ${ }^{1}$ (D), Giuseppe D’Ambrosio ${ }^{1}$, Giuseppe Romano ${ }^{4}$ (D), Francesco Clemenza ${ }^{4}$, \\ Silvia Sarullo ${ }^{2}$, Elio Venturini ${ }^{5}(\mathbb{D})$, Marco Gentile ${ }^{6}(\mathbb{D})$, Cinzia Nugara ${ }^{2,7}$ (D) Gabriella Iannuzzo ${ }^{6}$, \\ Antonello D'Andrea ${ }^{3}$, Carlo Vigorito ${ }^{1}$ and Filippo M. Sarullo ${ }^{2} \mathbb{D}$ \\ 1 Department of Translational Medical Sciences, Federico II University of Naples, 80131 Naples, Italy; \\ dilorenzoanna2@gmail.com (A.D.L.); alessandro.oliviero0695@gmail.com (A.O.); \\ francescopassaro1996@gmail.com (F.P.); roberta-calce@virgilio.it (R.C.); \\ alessandroparlato96@gmail.com (A.P.); kre.testa@gmail.com (C.T.); \\ giuseppe.dambrosio91@gmail.com (G.D.); vigorito@unina.it (C.V.) \\ 2 Cardiovascular Rehabilitation Unit, Buccheri La Ferla Fatebenefratelli Hospital, 90123 Palermo, Italy; \\ giuseppevit@hotmail.com (G.V.); silvia.sarullo@libero.it (S.S.); cinzianugara@gmail.com (C.N.); \\ sarullo.filippo@fbfpa.it (F.M.S.) \\ 3 Unit of Cardiology and Intensive Care, "Umberto I" Hospital, Viale San Francesco, \\ 84014 Nocera Inferiore (SA), Italy; pacmario@yahoo.it (M.P.); antonellodandrea@libero.it (A.D.) \\ 4 Cardiology Unit and Research Office, Department for the Treatment and Study of Cardiothoracic Diseases \\ and Cardiothoracic Transplantation IRCCS-ISMETT, 90127 Palermo, Italy; \\ romanogiuseppe81@gmail.com (G.R.); fclemenza@ismett.edu (F.C.) \\ 5 Cardiac Rehabilitation Unit, Azienda USL Toscana Nord-Ovest, Cecina Civil Hospital, \\ 57023 Cecina (LI), Italy; vent.elio@tin.it \\ 6 Department of Clinical Medicine and Surgery, Federico II University, 80131 Naples, Italy; \\ margenti@unina.it (M.G.); gabriella.iannuzzo@unina.it (G.I.) \\ 7 IRCCS Centro Neurolesi Bonino Pulejo Messina, University of Palermo, 90123 Palermo, Italy \\ * Correspondence: francesco.giallauria@unina.it; Tel.: +39-0817463519
}

Received: 15 May 2020; Accepted: 15 June 2020; Published: 17 June 2020

Abstract: Background: Heart rate recovery (HRR) is a marker of vagal tone, which is a powerful predictor of mortality in patients with cardiovascular disease. Sacubitril/valsartan (S/V) is a treatment for heart failure with reduced ejection fraction (HFrEF), which impressively impacts cardiovascular outcome. This study aims at evaluating the effects of S/V on HRR and its correlation with cardiopulmonary indexes in HFrEF patients. Methods: Patients with HFrEF admitted to outpatients' services were screened out for study inclusion. S/V was administered according to guidelines. Up-titration was performed every 4 weeks when tolerated. All patients underwent laboratory measurements, Doppler-echocardiography, and cardiopulmonary exercise stress testing (CPET) at baseline and at 12-month follow-up. Results: Study population consisted of $134 \mathrm{HFrEF}$ patients ( $87 \%$ male, mean age $57.9 \pm 9.6$ years). At 12 -month follow-up, significant improvement in left ventricular ejection fraction (from $28 \% \pm 5.8 \%$ to $31.8 \% \pm 7.3 \%, p<0.0001$ ), peak exercise oxygen consumption ( $\mathrm{VO}_{\text {2peak }}$ ) (from $15.3 \pm 3.7$ to $17.8 \pm 4.2 \mathrm{~mL} / \mathrm{kg} / \mathrm{min}, p<0.0001$ ), the slope of increase in ventilation over carbon dioxide output $\left(\mathrm{VE} / \mathrm{VCO}_{2}\right.$ slope $)$ (from $33.4 \pm 6.2$ to $30.3 \pm 6.5$, $p<0.0001$ ), and HRR (from $11.4 \pm 9.5$ to $17.4 \pm 15.1 \mathrm{bpm}, p=0.004$ ) was observed. Changes in HRR were significantly correlated to changes in $\mathrm{VE} / \mathrm{VCO}_{2 \text { slope }}(r=-0.330 ; p=0.003)$. After adjusting for potential confounding factors, multivariate analysis showed that changes in HRR were significantly associated to changes in $\mathrm{VE} / \mathrm{VCO}_{2 \text { slope }}$ (Beta $(\mathrm{B})=-0.975$, standard error $(\mathrm{SE})=0.364$, standardized Beta coefficient $(\mathrm{Bstd})=-0.304, p=0.009)$. $\mathrm{S} / \mathrm{V}$ showed significant reduction in exercise oscillatory 
ventilation (EOV) detection at CPET (28 EOV detected at baseline CPET vs. 9 EOV detected at 12-month follow-up, $p<0.001$ ). HRR at baseline CPET was a significant predictor of EOV at 12-month follow-up $(\mathrm{B}=-2.065, \mathrm{SE}=0.354, p<0.001)$. Conclusions: In HFrEF patients, $\mathrm{S} / \mathrm{V}$ therapy improves autonomic function, functional capacity, and ventilation. Whether these findings might translate into beneficial effects on prognosis and outcome remains to be elucidated.

Keywords: sacubitril/valsartan; heart failure; heart rate recovery; peak oxygen consumption; autonomic function; cardiopulmonary exercise stress testing

\section{Introduction}

Heart failure is characterized by autonomic dysfunction [1,2]. Heart rate recovery (HRR) after graded exercise stress testing is one of the commonly used indexes reflecting autonomic activity [1,2]. HRR is defined as the fall in heart rate during the first minute after exercise and is easily calculated as the difference in heart rate between the peak of exercise and one minute after exercise cessation. HRR is a marker of vagal tone, thanks to sympathetic deactivation and parasympathetic reactivation $[1,2]$. The first minute of post-exercise recovery is predominantly secondary to parasympathetic reactivation $[1,3-5]$. However, even though vagal-related HRR is parasympathetically mediated, previous studies suggest that it might represent independent aspects, but complementary information, with regard to parasympathetic function [6].

In patients with heart failure (HF), the total sympathetic nervous activity is augmented owing to loss of baroreflex sensitivity and to increased sympathetic afferent activity [7-9]. The sympathetic nervous system negatively impacts the cardiovascular system in HF; down-regulation of beta1-receptors and direct toxic effects on the myocardium contribute to cardiac remodeling and life-threatening arrhythmias [7-9].

Sacubitril/valsartan (S/V) is a treatment for HF with reduced ejection fraction (HFrEF), which impressively impact cardiovascular prognosis by reducing major adverse cardiovascular events such as cardiovascular mortality and sudden death [10]. Moreover, S/V significantly improves cardiopulmonary functional capacity and ventilatory parameters [11-14].

The present study aims at evaluating the effects of $\mathrm{S} / \mathrm{V}$ on autonomic function (as evaluated by $\mathrm{HRR}$ ) and its correlation with cardiopulmonary indexes in HFrEF patients.

\section{Experimental Section}

\subsection{Study Population}

Patients with HFrEF admitted to outpatients' services were screened for study inclusion. According to Italian reimbursement criteria, (1) symptomatic HF was defined as New York Heart Association (NYHA) class II-IV; (2) left ventricular ejection fraction (LVEF) $\leq 35 \%$; (3) previous treatment with maximal tolerated dose for at least 6 months of angiotensin-converting enzyme inhibitor (ACE-Inh) or angiotensin receptor blocker (ARB); (4) systolic blood pressure $\geq 100 \mathrm{mmHg}$; (5) serum potassium $\left(\mathrm{K}^{+}\right) \leq 5.4 \mathrm{mmol} / \mathrm{L}$, estimated glomerular filtration rate $\geq 30 \mathrm{~mL} / \mathrm{min} / 1.73 \mathrm{~m}^{2}$; (6) absence of severe liver disease (Child-Pugh C); and (7) no history of angioedema, S/V was prescribed when indicated. Patients were encouraged to undergo physical activity (according to patient's age, past habits, comorbidities, preferences, and goals) as indicated by European guidelines (at least $2.5 \mathrm{~h} /$ week of moderate intensity aerobic activity in multiple bouts each lasting $\geq 10 \mathrm{~min}, 5$ days/week) [15]. Exclusion criteria for study entry were as follows: HF hospitalization within 90 days before ambulatory evaluation, myocardial revascularization within 180 days before ambulatory evaluation, concomitant initiation of cardiac resynchronization therapy and/or percutaneous mitral valve treatment during study follow-up or in the previous 6 months, congenital heart disease, and inability to perform cardiopulmonary exercise 
stress testing (CPET). After inclusion, HFrEF patients were stratified according to angiotensin receptor neprilysin inhibitor (ARNI) dose $\left(\mathrm{ARNI}_{1}=24 / 26 \mathrm{mg}\right.$ bid, $\mathrm{ARNI}_{2}=49 / 51 \mathrm{mg}$ bid, and $\mathrm{ARNI}_{3}=97 / 103 \mathrm{mg}$ bid) [16]. Up-titration was performed every 4 weeks when tolerated. Changes in the dosage of diuretics were allowed during the study follow-up if deemed necessary. All patients underwent laboratory measurements, Doppler-echocardiography, and CPET at baseline and at 12-month follow-up.

The Institutional Research Review Boards of the Cardiovascular Rehabilitation Unit of Buccheri La Ferla Fatebenefratelli Hospital and of the Department for the Treatment and Study of Cardiothoracic Diseases and Cardiothoracic Transplantation IRCCS-ISMETT (IRRB/23/15; Palermo, Italy) approved this prospective, observational study. All patients provided informed consent.

\subsection{Laboratory Measurements}

N-terminal pro-brain natriuretic peptides (NT-proBNP) serum levels were detected at baseline and 12-month follow-up as previously described [17,18]. At baseline and after 12 months, fasting blood samples were collected between 08:00 and 09:00, avoiding blood sampling within $24 \mathrm{~h}$ of physical activity or CPET. NT-pro-BNP was determined with a sandwich immunoassay on an Elecsys 2010 (Roche diagnostics, Milano, Italy) with analytical range extended from 5 to $35.000 \mathrm{pg} / \mathrm{mL}$.

\subsection{Doppler Echocardiography}

Doppler echocardiography was performed a median of 1 day (range 0-3 days) after study enrollment and at 12-month follow-up. Measurements were obtained at least two times for an average. Standard views were recorded according to guidelines [19]. Indexing body size variables included body surface area $\left(B S A, \mathrm{~m}^{2}\right)$, body mass index $\left(B M I, \mathrm{~kg} / \mathrm{m}^{2}\right)$, and height $(\mathrm{cm})$. Indexed left atrial (LA) volume, indexed LV end-diastolic volume (LVEDV) and indexed LV end-systolic volume (LVESV), LV septal and posterior end-diastolic wall thickness, and LVEF were recorded. Non-indexed LA diameter, with $40 \mathrm{~mm}$ as the cut-off for normal, was also assessed [20,21]. Pulsed-wave Doppler echocardiography (before and with Valsalva maneuver) was used to explore mitral inflow from the apical four-chamber view [22]; and diastolic filling was categorized as normal (grade 0), impaired relaxation (grade 1), pseudonormal pattern (grade 2), or restrictive (grade 3) [23]. Global and regional myocardial function was investigated using Tissue Doppler imaging. The same physician, blinded to the patient allocation into the study protocol and unaware of the results of cardiopulmonary exercise stress testing (CPET), performed all Doppler-echocardiography studies.

\subsection{Cardiopulmonary Exercise Stress Testing}

All patients underwent incremental CPET on a bicycle ergometer as previously described [12]. Before each test, calibration of oxygen and carbon dioxide analyzers and of the flow mass sensor was performed using available precision gas mixtures and a $3 \mathrm{~L}$ syringe, respectively. In order to stabilize gas measurements, patients remained seated on the ergometer for at least $3 \mathrm{~min}$ before starting to pedal.

After a warm-up period ( $60 \mathrm{~s}$ at 0 Watts workload), a ramp protocol of $15 \mathrm{~W} / \mathrm{min}$ with pedaling kept constant at 55-65 revolutions/min was started and continued until exhaustion. Then, 12-lead electrocardiogram (ECG) was monitored continuously during the test, and cuff blood pressure was manually recorded every $2 \mathrm{~min}$. Breath-by-breath analysis for respiratory gas exchange measurements used computerized metabolic cart $\left(\mathrm{V}_{\max }{ }^{\circledR} 2900\right.$ metabolic cart, SensorMedics, Yorba Linda, CA, USA). $\mathrm{VO}_{2 \text { peak }}$ was recorded as the mean value of $\mathrm{VO}_{2}$ during the last $20 \mathrm{~s}$ of the test and expressed in millilitres per kilogram per minute. At the end of CPET, the primary reason for stopping was identified. Predicted $\mathrm{VO}_{2 \text { peak }}$ was determined by use of a sex-, age-, height-, and weight-adjusted and protocol-specific formula outlined by Wassermann et al. [24]. Two experienced reviewers (G.V. and F.M.S.) assessed ventilatory anaerobic threshold (VAT) with the V-slope method [25]. The ventilation (VE) versus carbon dioxide production $\left(\mathrm{VCO}_{2}\right)$ relationship was measured by plotting ventilation $(\mathrm{VE}, 1 / \mathrm{min})$ against carbon dioxide production $\left(\mathrm{VCO}_{2}, \mathrm{~L} / \mathrm{min}\right)$ obtained every $10 \mathrm{~s}$ of exercise $\left(\mathrm{VE} / \mathrm{VCO}_{2 \text { slope }}\right)$. The $\mathrm{VE} / \mathrm{VCO}_{2 \text { slope }}$ was calculated as a linear regression function, excluding the non-linear part of the 
relationship after the onset of acidotic drive to ventilation. Exercise oscillatory ventilation (EOV), a cyclic fluctuation of minute ventilation and expired gas kinetics occurring during exercise, was reported when identified [26]. The physician who will perform CPET will be unaware of the results of blood sampling and Doppler echocardiography.

\subsection{Statistics}

Descriptive statistics are given in terms of means \pm standard deviation or in percentage for nominal variables. $X^{2}$ test was used for contingency tables. Within-group changes in the reported variables were evaluated by the paired t-test or Wilcoxon signed rank test for non-normally distributed variables. Unpaired t-test and Mann-Whitney rank sum test were used for between-group comparisons. Differences between groups and changes over time within each group (time effect) as well as any interaction (different trends over time between groups) were assessed by two-way repeated measures analysis of variance (ANOVA). The bivariate correlations procedure was used to compute Pearson or Spearman correlation coefficients with the significance levels. Multiple linear regression analysis was used to test the association between changes in HRR and cardiopulmonary indexes. Models adjusted for potential confounding factors based upon a review of the relevant literature, including age, NYHA class, diabetes, atrial fibrillation, cardiac resynchronization therapy (CRT)/pacemaker, drug therapy (beta-blockers, ivabradine, and loop diuretics use, separately), NT-proBNP levels, maximal ARNI dose reached, and changes in $\mathrm{VO}_{2 \text { peak }}$ and changes in $\mathrm{VE} / \mathrm{VCO}_{2 \text { slope }}$. Logistic regression analysis was used for testing the association between HRR evaluated at baseline CPET and exercise oscillatory ventilation detected at 12 -months follow-up CPET. A $p$ value $<0.05$ was considered statistically significant. All analyses were performed by SPSS version 23.0 (SPSS Inc., Chicago, IL, USA).

\section{Results}

The study population consisted of $134 \mathrm{HFrEF}$ patients ( $87 \%$ male, mean age $57.9 \pm 9.6$ years). Baseline clinical and demographic characteristics of the study population and stratified by ARNI dose are given in Tables 1 and 2, respectively. Overall, mean LVEF was $28.1 \% \pm 5.9 \%$ and, at baseline, the majority of patients had NYHA class II (62\%) or class III (34\%), whereas no patients had NYHA class IV (Table 1). At 12-month follow-up, a significant improvement in NYHA class was observed: 104 patients (77.2\%) had NYHA class II and 15 patients (11.4\%) had NYHA class III (Table 1).

Major comorbidity was represented by hypertension (54\%), dyslipidemia (59\%), diabetes (34\%), chronic obstructive pulmonary disease (COPD) (16\%), and atrial fibrillation (16\%) (Table 1). At study entry, 6 patients $(4.5 \%)$ had pacemaker (PMK), 102 patients $(76 \%)$ had PMK plus implantable cardioverter-defibrillator (ICD), and 23 patients (17\%) had cardiac resynchronization therapy (CRT) devices (Table 1). At 12-month follow-up, 103 patients (77\%) had PMK-ICD and a total of 25 (18\%) patients had CRT devices. Forty-four patients were active smokers (Table 1). 
Table 1. Clinical and demographic characteristics of the study population at baseline and 12-month follow-up.

\begin{tabular}{|c|c|c|c|}
\hline & Baseline & 12-Month Follow-Up & \\
\hline Age (Years) & $57.9 \pm 9.6$ & & \\
\hline Gender (Male) (\%) & 87 & & \\
\hline BMI $\left(\mathrm{kg} / \mathrm{m}^{2}\right)$ & $28.3 \pm 4.5$ & $28.2 \pm 4.6$ & 0.935 \\
\hline NT-proBNP $(\mathrm{pg} / \mathrm{mL})$ & $1443.2 \pm 1323$ & $1041.2 \pm 1171.3$ & 0.015 \\
\hline eGFR (MDRD) $\left(\mathrm{mL} / \mathrm{min} / 1.73 \mathrm{~m}^{2}\right)$ & $73.1 \pm 36.3$ & $76.8 \pm 38.8$ & 0.518 \\
\hline Haemoglobin $(\mathrm{g} / \mathrm{dL})$ & $12.8 \pm 1.4$ & $14.2 \pm 2.5$ & 0.388 \\
\hline $\mathrm{Na}^{+}(\mathrm{mEq} / \mathrm{L})$ & $139.2 \pm 2.6$ & $140 \pm 2.3$ & 0.06 \\
\hline $\mathrm{K}^{+}(\mathrm{mEq} / \mathrm{L})$ & $4.2 \pm 0.5$ & $4.3 \pm 0.5$ & 0.691 \\
\hline $\mathrm{SBP}(\mathrm{mmHg})$ & $117.1 \pm 16.9$ & $102.9 \pm 13.4$ & $<0.0001$ \\
\hline $\mathrm{DBP}(\mathrm{mmHg})$ & $71.8 \pm 9.7$ & $64.2 \pm 6.9$ & $<0.0001$ \\
\hline HR (bpm) & $68.2 \pm 11.5$ & $67.1 \pm 8.9$ & 0.435 \\
\hline NYHA Class I & $5(4 \%)$ & $15(11.4 \%) * *$ & $<0.0001$ \\
\hline NYHA Class II & $82(62 \%)$ & $104(77.2 \%)$ & \\
\hline NYHA Class III & $46(34 \%)$ & $15(11.4 \%)$ & \\
\hline NYHA Class IV & & & \\
\hline Pacemaker $(n, \%)$ & $6(4.5 \%)$ & & \\
\hline CRT $(n, \%)$ & $23(17 \%)$ & & \\
\hline PMK-ICD $(n, \%)$ & $102(76 \%)$ & & \\
\hline Hypertension $(n, \%)$ & $72(54 \%)$ & & \\
\hline Diabetes $(n, \%)$ & $45(34 \%)$ & & \\
\hline Dyslipidemia $(n, \%)$ & $78(59 \%)$ & & \\
\hline Atrial Fibrillation $(n, \%)$ & $22(16 \%)$ & & \\
\hline $\operatorname{COPD}(n, \%)$ & $21(16 \%)$ & & \\
\hline Active Smokers & $58(44 \%)$ & & \\
\hline \multicolumn{4}{|l|}{ Drug therapy } \\
\hline Beta-Blockers $(n, \%)$ & $125(93 \%)$ & $125(93 \%)$ & 1.00 \\
\hline ACE-Inhibitors * $(n, \%)$ & $81(60 \%)$ & & \\
\hline $\mathrm{ARBs}^{*}(n, \%)$ & $29(21 \%)$ & & \\
\hline Loop Diuretics $(n, \%)$ & $111(83 \%)$ & $103(77 \%)$ & 0.04 \\
\hline Loop Diuretics Dose (mg/day) & $87 \pm 105$ & $80 \pm 97$ & 0.586 \\
\hline $\operatorname{MRA}(n, \%)$ & $110(82 \%)$ & $109(81 \%)$ & 0.533 \\
\hline MRA dose (mg/day) & $26 \pm 12$ & $24 \pm 9$ & 0.174 \\
\hline Ivabradine $(n, \%)$ & $25(19 \%)$ & $25(19 \%)$ & 1.00 \\
\hline Statin $(n, \%)$ & $97(72 \%)$ & $95(70 \%)$ & 1.00 \\
\hline $\mathrm{ARNI}_{1}(n, \%)$ & $90(67.2 \%)$ & $39(29.1 \%)^{* *}$ & $<0.0001$ \\
\hline $\mathrm{ARNI}_{2}(n, \%)$ & $40(31.3 \%)$ & $48(35.8 \%)$ & \\
\hline $\mathrm{ARNI}_{3}(n, \%)$ & $2(1.5 \%)$ & $47(35.1 \%)$ & \\
\hline
\end{tabular}

Captions: ACE-inhibitors: angiotensin converting enzyme inhibitors ( ${ }^{*}$ prior to ARNI therapy); ARBs: angiotensin receptor blockers (* prior to ARNI therapy); $\mathrm{ARNI}_{1}$ : angiotensin receptor neprilysin inhibitor (ARNI) dose of 24/26 mg bid at baseline; ARNI2: ARNI dose of 49/51 mg bid at baseline; ARNI $_{3}$ : ARNI dose of 97/103 mg bid at baseline; BMI: body mass index; COPD: chronic obstructive pulmonary disease; CRT: cardiac resynchronization therapy; DBP: diastolic blood pressure (at the time of cardiopulmonary exercise stress testing (CPET)); eGFR (MDRD): estimated glomerular filtration rate; HR: heart rate; ICD: implantable cardioverter-defibrillator; MRA: mineral corticoid receptor antagonists; NT-proBNP: N-terminal pro-brain natriuretic peptide; NYHA: New York Heart Association; PMK: pacemaker; SBP: systolic blood pressure (at the time of CPET). ${ }^{* *} \mathrm{X}^{2}$ test was used for contingency tables. 
Table 2. Clinical and demographic characteristics of the study population stratified by ARNI dose.

\begin{tabular}{|c|c|c|c|c|c|c|}
\hline & \multicolumn{2}{|c|}{$\mathrm{ARNI}_{1}$} & \multicolumn{2}{|c|}{$\mathrm{ARNI}_{2}$} & \multicolumn{2}{|c|}{$\mathrm{ARNI}_{3}$} \\
\hline & $\begin{array}{l}\text { Baseline } \\
(n=90)\end{array}$ & $\begin{array}{l}\text { 12-Month } \\
\text { Follow-Up } \\
(n=39)\end{array}$ & $\begin{array}{l}\text { Baseline } \\
(n=40)\end{array}$ & $\begin{array}{c}\text { 12-Month } \\
\text { Follow-Up } \\
(n=48)\end{array}$ & $\begin{array}{l}\text { Baseline } \\
(n=2)\end{array}$ & $\begin{array}{c}\text { 12-Month } \\
\text { Follow-Up } \\
(n=47)\end{array}$ \\
\hline BMI $\left(\mathrm{kg} / \mathrm{m}^{2}\right)$ & $27.2 \pm 3.9$ & $26.9 \pm 4.0$ & $30.4 \pm 5.1$ & $28.8 \pm 4.7$ & $31.4 \pm 2.3$ & $28.6 \pm 4.9$ \\
\hline NT-proBNP (pg/mL) & $1450.15 \pm 1466.52$ & $1343.55 \pm 1373.42$ & $1390.70 \pm 991.90$ & $1151.68 \pm 1303.11$ & $2146 \pm 11.49$ & $673.3 \pm 704.7^{*}$ \\
\hline eGFR (MDRD) $\left(\mathrm{mL} / \mathrm{min} / 1.73 \mathrm{~m}^{2}\right)$ & $73.3 \pm 39.2$ & $69.3 \pm 23.7$ & $72.5 \pm 31.1$ & $82.2 \pm 55.0$ & $77.5 \pm 15.9$ & $75.6 \pm 13.9$ \\
\hline $\mathrm{K}^{+}(\mathrm{mEq} / \mathrm{L})$ & $4.2 \pm 0.43$ & $4.4 \pm 0.60$ & $4.3 \pm 0.54$ & $4.2 \pm 0.36$ & $4.5 \pm 0.32$ & $4.3 \pm 0.53$ \\
\hline $\mathrm{SBP}(\mathrm{mmHg})$ & $114.9 \pm 14.5$ & $96.9 \pm 11.5^{*}$ & $121.8 \pm 20.2$ & $102.9 \pm 12.0 *$ & $122.5 \pm 31.8$ & $107.9 \pm 14.5$ \\
\hline DBP (mmHg) & $71.3 \pm 9.6$ & $62.4 \pm 6.7^{*}$ & $72.7 \pm 10.2$ & $63.9 \pm 6.6^{*}$ & $77.5 \pm 3.5$ & $65.9 \pm 7.2 *$ \\
\hline HR (bpm) & $67.8 \pm 10.5$ & $67.3 \pm 7.016$ & $68.0 \pm 12.5$ & $67.4 \pm 8.6$ & $86.5 \pm 26.2$ & $66.6 \pm 10.9 *$ \\
\hline
\end{tabular}

Captions: ${ }^{*} p<0.001$ vs. baseline; $\mathrm{ARNI}_{1}$ : angiotensin receptor neprilysin inhibitor (ARNI) dose of 24/26 mg bid at baseline; ARNI2: ARNI dose of 49/51 mg bid at baseline; ARNI 3 : ARNI dose of $97 / 103 \mathrm{mg}$ bid at baseline; BMI: body mass index; DBP: diastolic blood pressure (at the time of cardiopulmonary exercise stress testing (CPET)); eGFR (MDRD): estimated glomerular filtration rate; HR: heart rate; NT-proBNP: N-terminal pro-brain natriuretic peptide; SBP: systolic blood pressure (at the time of CPET).

Clinical and demographic characteristics of the study population stratified by ARNI dose are given in Table 3 At study entry, 90 (67.2\%) HFrEF patients were taking $\mathrm{ARNI}_{1}$ dose, 40 (31.3\%) HFrEF patients were taking $\mathrm{ARNI}_{2}$ dose, and $2(1.5 \%) \mathrm{HFrEF}$ patients were taking $\mathrm{ARNI}_{3}$ dose; whereas at 12-month follow-up, 39 (29.1\%) HFrEF patients were taking $\mathrm{ARNI}_{1}$ dose, 48 (35.8\%) HFrEF patients were taking $\mathrm{ARNI}_{2}$ dose, and 47 (35.1\%) HFrEF patients were taking $\mathrm{ARNI}_{3}$ dose.

Table 3. Baseline and 12-month follow-up Doppler-echocardiography parameters in overall population.

\begin{tabular}{cccc}
\hline Doppler-Echocardiography Parameters & Baseline & $\begin{array}{c}\text { 12-Month } \\
\text { Follow-Up }\end{array}$ & $p$-Value \\
\hline LVEDD (mm) & $63.1 \pm 6.7$ & $63.6 \pm 6.5$ & 0.701 \\
LVEDV (mL) & $216.2 \pm 56.3$ & $208.7 \pm 59.1$ & 0.482 \\
LVEDVi (mL/m²) & $112.2 \pm 27.9$ & $112.9 \pm 25.8$ & 0.897 \\
LVEF (\%) & $28 \pm 5.8$ & $31.8 \pm 7.3$ & $<0.0001$ \\
LAV (mL) & $96.7 \pm 35.8$ & $86.1 \pm 29.3$ & 0.098 \\
LAVi (mL/m $\left.{ }^{2}\right)$ & $49.6 \pm 17.9$ & $45.6 \pm 14.7$ & 0.226 \\
E-Wave (cm/s) & $0.92 \pm 0.43$ & $0.85 \pm 0.42$ & 0.434 \\
A-Wave (cm/s) & $0.67 \pm 0.31$ & $0.79 \pm 0.31$ & 0.094 \\
E/A Ratio & $1.53 \pm 1.4$ & $1.01 \pm 0.74$ & 0.02 \\
Deceleration Time (ms) & $175.9 \pm 53.8$ & $197.2 \pm 59.4$ & 0.113 \\
E' (cm/s) & $0.05 \pm 0.02$ & $0.06 \pm 0.02$ & 0.047 \\
E/E' ratio & $15.1 \pm 6.8$ & $13.4 \pm 7.0$ & 0.298 \\
TAPSE (mm) & $19.5 \pm 4.2$ & $19.3 \pm 3.9$ & 0.398
\end{tabular}

Captions: A-wave: peak velocity flow in late diastole caused by atrial contraction; E-wave: peak velocity blood flow from left ventricular relaxation in early diastole; $E^{\prime}$-wave: early diastolic myocardial relaxation; LAV: left atrium volume; LAVi: left atrium indexed volume; LVEDD: left ventricular end-diastolic diameter; LVEDV: left ventricular end-diastolic volume; LVEDVi: left ventricular end-diastolic indexed volume; LVEF: left ventricular ejection fraction; TAPSE: tricuspid annular plane excursion.

Baseline and 12-month follow-up Doppler-echocardiography parameters in overall population and stratified by ARNI dose are reported in Tables 3 and 4, respectively. A significant improvement in LVEF was observed at 12-month follow-up (from $28 \% \pm 5.8 \%$ to $31.8 \% \pm 7.3 \%, p<0.0001$ ) (Table 3). LVEF improvement was greater among patients treated with higher ARNI doses (from $28.4 \% \pm 6.2 \%$ to $29.2 \% \pm 7.2 \%, p=0.415$ in $\mathrm{ARNI}_{1}$ group; from $27.4 \% \pm 6.4 \%$ to $32.1 \% \pm 6.5 \%, p=0.006$ in $\mathrm{ARNI}_{2}$ group; and from $28.2 \% \pm 4.9 \%$ to $33.8 \% \pm 8.0 \%, p=0.001$ in $\mathrm{ARNI}_{3}$ group). 
Table 4. Baseline and 12-month follow-up Doppler-echocardiography parameters in overall population stratified by ARNI dose.

\begin{tabular}{|c|c|c|c|c|c|c|}
\hline & \multicolumn{2}{|c|}{$\mathrm{ARNI}_{1}$} & \multicolumn{2}{|c|}{$\mathrm{ARNI}_{2}$} & \multicolumn{2}{|c|}{$\mathrm{ARNI}_{3}$} \\
\hline & $\begin{array}{l}\text { Baseline } \\
(n=90)\end{array}$ & $\begin{array}{c}\text { 12-Month } \\
\text { Follow-Up } \\
\quad(n=39)\end{array}$ & $\begin{array}{l}\text { Baseline } \\
(n=40)\end{array}$ & $\begin{array}{c}\text { 12-Month } \\
\text { Follow-Up } \\
\quad(n=48)\end{array}$ & $\begin{array}{l}\text { Baseline } \\
(n=2)\end{array}$ & $\begin{array}{c}\text { 12-Month } \\
\text { Follow-Up } \\
(n=47)\end{array}$ \\
\hline LVEDD (mm) & $62.8 \pm 6.4$ & $64.3 \pm 6.1$ & $63.8 \pm 7.7$ & $65.1 \pm 5.4$ & $68.0 \pm 2.8$ & $61.4 \pm 7.6$ \\
\hline LVEDV (mL) & $210.9 \pm 57.7$ & $210.7 \pm 53.1$ & $224.3 \pm 51.5$ & $213.6 \pm 67.2$ & $281.0 \pm 41$ & $203.3 \pm 64.1$ \\
\hline $\operatorname{LVEDVi~}\left(\mathrm{mL} / \mathrm{m}^{2}\right)$ & $112.7 \pm 28.4$ & $110.9 \pm 25.7$ & $107.9 \pm 26.6$ & $115.9 \pm 21.4$ & $140.5 \pm 13.4$ & $113.9 \pm 29.9$ \\
\hline $\operatorname{LVEF}(\%)$ & $28.4 \pm 6.2$ & $29.2 \pm 7.2$ & $27.4 \pm 6.4$ & $32.1 \pm 6.5 *$ & $28.2 \pm 4.9$ & $33.8 \pm 8.0$ \\
\hline LAV (mL) & $92.7 \pm 35.0$ & $90.8 \pm 27.6$ & $106.4 \pm 38.4$ & $86.7 \pm 37.1$ & $107.0 \pm 18.4$ & $79.8 \pm 27.4$ \\
\hline LAVi $\left(\mathrm{mL} / \mathrm{m}^{2}\right)$ & $49.1 \pm 19.2$ & $48.9 \pm 16.3$ & $50.9 \pm 14.7$ & $44.3 \pm 14.4$ & $53 \pm 4.2$ & $41.6 \pm 12.6$ \\
\hline E-Wave $(\mathrm{cm} / \mathrm{s})$ & $0.90 \pm 0.43$ & $0.97 \pm 0.46$ & $1.01 \pm 0.44$ & $0.8 \pm 0.23$ & $0.65 \pm 0.03$ & $0.72 \pm 0.45$ \\
\hline A-Wave $(\mathrm{cm} / \mathrm{s})$ & $0.64 \pm 0.30$ & $0.71 \pm 0.28$ & $0.75 \pm 0.33$ & $0.85 \pm 0.42$ & $0.85 \pm 0.04$ & $0.83 \pm 0.22$ \\
\hline E/A Ratio & $1.6 \pm 1.5$ & $1.36 \pm 1.44$ & $0.89 \pm 0.55$ & $0.94 \pm 0.31$ & $0.76 \pm 0.035$ & $0.87 \pm 0.34$ \\
\hline Deceleration Time (ms) & $172.5 \pm 55.1$ & $191.8 \pm 55.9$ & $193.2 \pm 47.1$ & $185.0 \pm 55.9$ & $143.5 \pm 61.5$ & $212.4 \pm 67.9$ \\
\hline$E^{\prime}(\mathrm{cm} / \mathrm{s})$ & $0.05 \pm 0.02$ & $0.06 \pm 0.01$ & $0.06 \pm 0.01$ & $0.06 \pm 0.02$ & $0.05 \pm 0.01$ & $0.06 \pm 0.02$ \\
\hline $\mathrm{E} / \mathrm{E}^{\prime}$ ratio & $15.2 \pm 7.5$ & $13.2 \pm 4.6$ & $15.3 \pm 4.6$ & $13.5 \pm 5.7$ & $13.3 \pm 3.3$ & $13.6 \pm 10.4$ \\
\hline TAPSE (mm) & $19.0 \pm 4.1$ & $17.6 \pm 3.1$ & $20.8 \pm 4.7$ & $19.6 \pm 3.3$ & $18.5 \pm 0.7$ & $20.0 \pm 3.3$ \\
\hline
\end{tabular}

Captions: ${ }^{*} p=0.01$ vs. baseline; A-wave: peak velocity flow in late diastole caused by atrial contraction; $\mathrm{ARNI}_{1}$ : angiotensin receptor neprilysin inhibitor (ARNI) dose of 24/26 mg bid; ARNI2: ARNI dose of 49/51 mg bid; $\mathrm{ARNI}_{3}$ : ARNI dose of 97/103 mg bid; E-wave: peak velocity blood flow from left ventricular relaxation in early diastole; E'-wave: early diastolic myocardial relaxation; LAV: left atrium volume; LAVi: left atrium indexed volume; LVEDD: left ventricular end-diastolic diameter; LVEDV: left ventricular end-diastolic volume; LVEDVi: left ventricular end-diastolic indexed volume; LVEF: left ventricular ejection fraction; TAPSE: tricuspid annular plane excursion.

Baseline and 12-month follow-up CPET parameters in the overall population and stratified by ARNI dose are reported in Tables 5 and 6, respectively. Overall, S/V therapy exerted beneficial effects on cardiopulmonary functional capacity (Table 5). Significant changes in $\mathrm{VO}_{2 \text { peak }}$ and predicted $\mathrm{VO}_{2 \text { peak }}$ (\%) were observed at 12-month follow-up (from $15.3 \pm 3.7$ to $17.8 \pm 4.2 \mathrm{~mL} / \mathrm{kg} / \mathrm{min}, p<0.0001$; and from $56.4 \% \pm 13.9 \%$ to $64.8 \% \pm 17.8 \%, p<0.0001$, respectively) (Table 5). Mean $\mathrm{VO}_{2 \text { peak }}(\mathrm{mL} / \mathrm{kg} / \mathrm{min}$ ) values according to ARNI dose at baseline and 12-month follow-up are shown in Figure 1. Compared with the lowest ARNI treatment dose, HFrEF patients receiving the higher ARNI dose (97/103 mg bid) had greater $\mathrm{VO}_{2 \text { peak }}$ improvement $(p<0.0001)$ (Figure 1). Compared with baseline, significant improvement in other cardiopulmonary functional capacity parameters were observed at 12-month follow-up: maximal workload and $\Delta \mathrm{VO} 2 / \Delta$ work significantly improved from $72.8 \pm 25.1$ to $90.5 \pm 28.1$ watts, $p<0.0001$, and from $9.2 \pm 1.6$ to $10.2 \pm 1.5 \mathrm{~mL} / \mathrm{min} /$ watt, $p<0.0001$, respectively (Table 5 ).

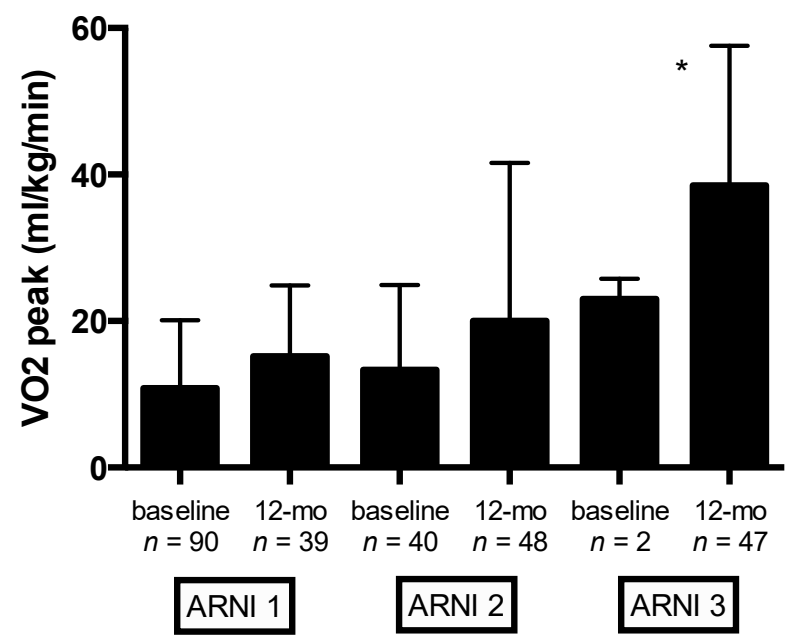

Figure 1. Baseline and 12-month follow-up values of $\mathrm{VO} 2$ peak $(\mathrm{mL} / \mathrm{Kg} / \mathrm{min})$ stratified by ARNI dose. Captions: angiotensin receptor neprilysin inhibitor (ARNI) dose $\left(\mathrm{ARNI}_{1}=24 / 26 \mathrm{mg}\right.$ bid, $\mathrm{ARNI}_{2}=49 / 51 \mathrm{mg}$ bid, and $\mathrm{ARNI}_{3}=97 / 103 \mathrm{mg}$ bid); peak oxygen consumption $\left(\mathrm{VO} 2_{\text {peak }}\right) .{ }^{*} p<0.0001$ (ANOVA). 
Table 5. Baseline and 12-month follow-up cardiopulmonary parameters in overall population.

\begin{tabular}{|c|c|c|c|}
\hline CPET Parameters & Baseline & 12-Month Follow-Up & $p$ Value \\
\hline $\mathrm{VO}_{2 \text { peak }}(\mathrm{mL} / \mathrm{kg} / \mathrm{min})$ & $15.3 \pm 3.7$ & $17.8 \pm 4.2$ & $<0.0001$ \\
\hline Predicted $\mathrm{VO}_{2 \text { peak }}(\%)$ & $56.4 \pm 13.9$ & $64.8 \pm 17.8$ & $<0.0001$ \\
\hline Watt Max (W) & $72.8 \pm 25.1$ & $90.5 \pm 28.1$ & $<0.0001$ \\
\hline $\mathrm{VE} / \mathrm{VCO}_{2 \text { slope }}$ & $33.4 \pm 6.2$ & $30.3 \pm 6.5$ & $<0.0001$ \\
\hline RER & $1.14 \pm 0.12$ & $1.14 \pm 0.15$ & 0.836 \\
\hline HR Rest (bpm) & $72.4 \pm 10.8$ & $68.9 \pm 12.1$ & 0.329 \\
\hline HR Peak (bpm) & $106.6 \pm 18.2$ & $110.9 \pm 20.6$ & 0.001 \\
\hline HRR (bpm) & $11.4 \pm 9.5$ & $17.4 \pm 15.1$ & 0.004 \\
\hline AT-VO $2(\mathrm{~mL} / \mathrm{kg} / \mathrm{min})$ & $11.4 \pm 2.9$ & $12.1 \pm 3.6$ & 0.015 \\
\hline $\mathrm{AT}-\mathrm{VO}_{2}(\%)$ & $40.9 \pm 11.5$ & $55.5 \pm 84.6$ & 0.173 \\
\hline AT-Watt (W) & $56.1 \pm 24.8$ & $58.5 \pm 22.1$ & 0.536 \\
\hline $\mathrm{O}_{2}$-Pulse (mL/beat) & $11.4 \pm 3$ & $13.7 \pm 4.6$ & $<0.0001$ \\
\hline$\Delta \mathrm{VO}_{2} / \Delta$ work $(\mathrm{mL} / \mathrm{min} /$ watt $)$ & $9.2 \pm 1.6$ & $10.2 \pm 1.5$ & $<0.0001$ \\
\hline Peak Ventilation (L/min) & $48.1 \pm 12.3$ & $61.4 \pm 18.9$ & $<0.0001$ \\
\hline TIDAL Volume (mL/kg) & $1.5 \pm 0.38$ & $2.2 \pm 3.7$ & 0.114 \\
\hline Respiratory Rate (breaths/min) & $30.8 \pm 6.3$ & $33.6 \pm 7.4$ & $<0.0001$ \\
\hline
\end{tabular}

Captions: AT: anaerobic threshold; RER: respiratory exchange ratio; VE: ventilation; $\mathrm{VE} / \mathrm{VCO}_{2 s l o p e}$ : the slope of increase in ventilation over carbon dioxide output; $\mathrm{VO}_{2}$ : oxygen consumption; $\mathrm{HR}$ : heart rate; HRR: heart rate recovery; CPET: cardiopulmonary exercise stress testing.

Table 6. Baseline and 12-month follow-up cardiopulmonary parameters in study population stratified by ARNI dose.

\begin{tabular}{|c|c|c|c|c|c|c|}
\hline & \multicolumn{2}{|c|}{$\mathrm{ARNI}_{1}$} & \multicolumn{2}{|c|}{$\mathrm{ARNI}_{2}$} & \multicolumn{2}{|c|}{$\mathrm{ARNI}_{3}$} \\
\hline & $\begin{array}{l}\text { Baseline } \\
(n=90)\end{array}$ & $\begin{array}{l}\text { 12-Month } \\
\text { Follow-Up } \\
\quad(n=39)\end{array}$ & $\begin{array}{l}\text { Baseline } \\
(n=40)\end{array}$ & $\begin{array}{l}\text { 12-Month } \\
\text { Follow-Up } \\
(n=48)\end{array}$ & $\begin{array}{l}\text { Baseline } \\
(n=2)\end{array}$ & $\begin{array}{l}\text { 12-Month } \\
\text { Follow-Up } \\
\quad(n=47)\end{array}$ \\
\hline $\mathrm{VO}_{2 \text { peak }}(\mathrm{mL} / \mathrm{kg} / \mathrm{min})$ & $15.3 \pm 3.7$ & $16.5 \pm 4.2$ & $14.8 \pm 3.6$ & $17.5 \pm 4.7^{* *}$ & $14.2 \pm 2.7$ & $18.9 \pm 4.7$ \\
\hline Predicted $\mathrm{VO}_{2 \text { peak }}(\%)$ & $56.4 \pm 13.9$ & $62.5 \pm 14.4^{*}$ & $54.0 \pm 14.5$ & $65.4 \pm 16.0^{* *}$ & $50.5 \pm 21.9$ & $68.7 \pm 19.1$ \\
\hline Watt Max (W) & $72.8 \pm 25.1$ & $80.6 \pm 31.6$ & $78.2 \pm 24.9$ & $86.8 \pm 28.9$ & $63.0 \pm 7.0$ & $102.8 \pm 30.7$ \\
\hline $\mathrm{VE} / \mathrm{VCO}_{2 \text { slope }}$ & $33.4 \pm 6.2$ & $32.2 \pm 5.9$ & $32.3 \pm 5.6$ & $31.3 \pm 7.1$ & $38.1 \pm 3.8$ & $28.9 \pm 5.2^{* *}$ \\
\hline RER & $1.14 \pm 0.12$ & $1.13 \pm 0.09$ & $1.13 \pm 0.07$ & $1.16 \pm 0.11$ & $1.07 \pm 0.10$ & $1.15 \pm 0.10$ \\
\hline HR Rest (bpm) & $72.4 \pm 10.8$ & $68.7 \pm 7.2$ & $70.9 \pm 10.9$ & $68.3 \pm 9.1$ & $80.5 \pm 27.6$ & $69.4 \pm 10.1$ \\
\hline HR Peak (bpm) & $106.6 \pm 18.2$ & $106.7 \pm 19.9$ & $106.3 \pm 19.3$ & $110.3 \pm 15.9$ & $126.0 \pm 26.9$ & $115.1 \pm 22.1$ \\
\hline $\operatorname{HRR}(\mathrm{bpm})$ & $11.4 \pm 9.5$ & $14.1 \pm 10.9$ & $12.2 \pm 11.2$ & $15.0 \pm 9.9$ & $23.0 \pm 2.8$ & $18.7 \pm 10.0$ \\
\hline AT-VO $2(\mathrm{~mL} / \mathrm{kg} / \mathrm{min})$ & $11.4 \pm 2.9$ & $12.3 \pm 2.9$ & $11.4 \pm 2.3$ & $12.2 \pm 3.7$ & $10.9 \pm 2.7$ & $13.0 \pm 2.9$ \\
\hline $\mathrm{AT}-\mathrm{VO}_{2}(\%)$ & $40.9 \pm 11.5$ & $45.7 \pm 13.5$ & $46.0 \pm 13.4$ & $45.8 \pm 13.4$ & $41.0 \pm 11.3$ & $50.2 \pm 12.5^{* *}$ \\
\hline AT-Watt (W) & $56.1 \pm 24.8$ & $57.2 \pm 25.6$ & $59.5 \pm 19.7$ & $55.1 \pm 19.2$ & $48.0 \pm 21.3$ & $67.3 \pm 22.9 * *$ \\
\hline $\mathrm{O}_{2}$-Pulse (mL/beat) & $11.4 \pm 3.0$ & $12.2 \pm 3.5$ & $12.6 \pm 3.2$ & $13.2 \pm 3.8$ & $10.2 \pm 2.4$ & $14.4 \pm 3.8^{*}$ \\
\hline$\Delta \mathrm{VO}_{2} / \Delta$ work $(\mathrm{mL} / \mathrm{min} /$ watt $)$ & $9.2 \pm 1.6$ & $9.6 \pm 1.8$ & $9.0 \pm 1.7$ & $10.1 \pm 1.5^{* *}$ & $9.5 \pm 0.6$ & $10.1 \pm 1.5$ \\
\hline Peak Ventilation (L/min) & $45.8 \pm 10.6$ & $55.0 \pm 17.9^{* *}$ & $52.7 \pm 14.7$ & $56.4 \pm 15.0$ & $47.2 \pm 9.5$ & $63.6 \pm 18.8^{* *}$ \\
\hline TIDAL volume $(\mathrm{mL} / \mathrm{kg})$ & $1.5 \pm 0.38$ & $1.7 \pm 0.56 *$ & $1.7 \pm 0.47$ & $1.6 \pm 0.44$ & $1.6 \pm 0.17$ & $1.9 \pm 0.5$ \\
\hline Respiratory Rate (breaths/min) & $30.8 \pm 6.3$ & $33.2 \pm 6.9$ & $31.7 \pm 6.5$ & $34.7 \pm 6.0 *$ & $30.0 \pm 2.8$ & $32.9 \pm 7.0^{* *}$ \\
\hline
\end{tabular}

Captions: ${ }^{*} p<0.05,{ }^{* *} p<0.001$ vs. baseline; $\mathrm{ARNI}_{1}$ : angiotensin receptor neprilysin inhibitor (ARNI) dose of 24/26 $\mathrm{mg}$ bid; ARNI2: ARNI dose of 49/51 mg bid; $\mathrm{ARNI}_{3}$ : ARNI dose of 97/103 mg bid; AT: anaerobic threshold; RER: respiratory exchange ratio; VE: ventilation; VE/VCO2slope: the slope of increase in ventilation over carbon dioxide output; VO2: oxygen consumption; HR: heart rate; HRR: heart rate recovery.

Moreover, significant improvement in VE/ $\mathrm{VCO}_{2 \text { slope }}$ was observed at 12-month follow-up (from $33.4 \pm 6.2$ to $30.3 \pm 6.5, p<0.0001$ ) (Table 5). Mean changes in $\mathrm{VE} / \mathrm{VCO}_{2 \text { slope }}$ according to ARNI dose at baseline and 12-month follow-up are shown in Figure 2. Compared with the lowest ARNI treatment dose, HFrEF patients receiving the higher ARNI dose (97/103 mg bid) had greater VE/VCO ${ }_{2 s l o p e}$ improvement $(p<0.0001)$ (Figure 2). Notably, significant improvement in other ventilatory parameters was observed at 12-month follow-up: peak ventilation and respiratory rate significantly increase from baseline (from $48.1 \pm 12.3$ to $61.4 \pm 18.9 \mathrm{~L} / \mathrm{min}, p<0.0001$; and from $30.8 \pm 6.3$ to $33.6 \pm 7.4, p<0.0001$, respectively) (Table 5). In addition, HFrEF patients treated with $\mathrm{S} / \mathrm{V}$ showed a significant reduction in EOV detection at CPET (28 EOV detected at baseline CPET vs. 9 EOV detected at 12-month follow-up, $p<0.001)$. HRR at baseline CPET was a significant predictor of EOV at 12-month follow-up $(\mathrm{B}=-2.065$, $\mathrm{SE}=0.354, p<0.001)$. 

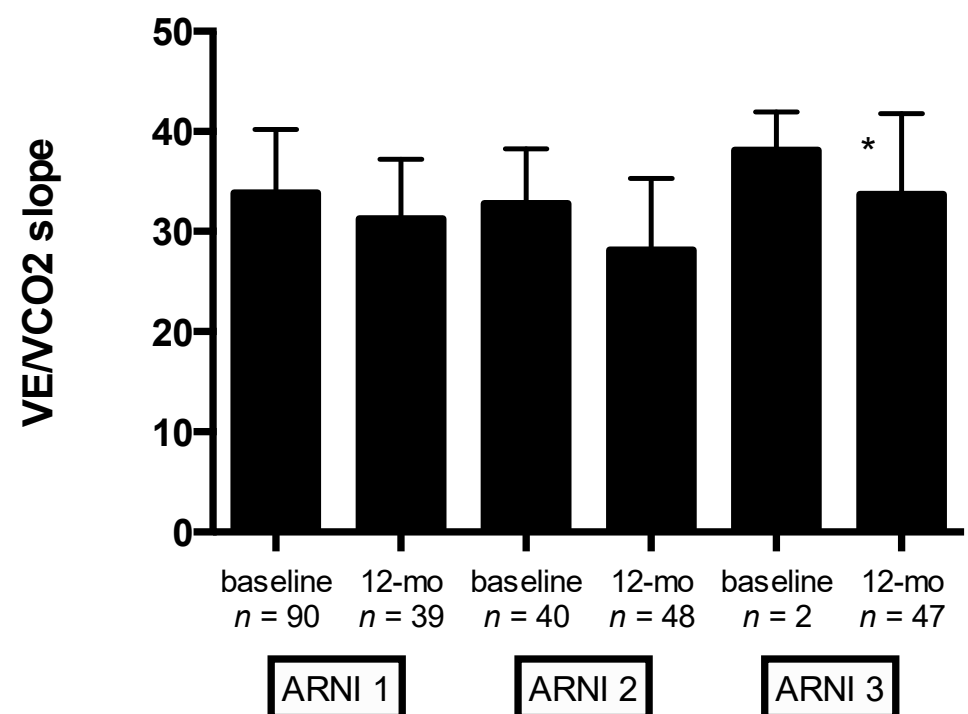

Figure 2. Baseline and 12-month follow-up values of $\mathrm{VE} / \mathrm{VCO}_{2 \text { slope }}$ stratified by ARNI dose. Captions: angiotensin receptor neprilysin inhibitor (ARNI) dose $\left(\mathrm{ARNI}_{1}=24 / 26 \mathrm{mg}\right.$ bid, $\mathrm{ARNI}_{2}=49 / 51 \mathrm{mg}$ bid, and $\mathrm{ARNI}_{3}=97 / 103 \mathrm{mg}$ bid); the slope of increase in ventilation over carbon dioxide output (VE/VCO2slope); ${ }^{*} p<0.0001$ (ANOVA).

Significant changes in autonomic function (as evaluated by HRR) were observed at 12-month follow-up (from $11.4 \pm 9.5$ to $17.4 \pm 15.1 \mathrm{bpm}, p=0.004$ ) (Table 5). Mean changes in HRR stratified by ARNI dose at baseline and 12-month follow-up are shown in Figure 3. Compared with the lowest ARNI treatment dose, HFrEF patients receiving the higher ARNI dose (97/103 mg bid) had greater HRR improvement $(p=0.009)$ (Figure 3).

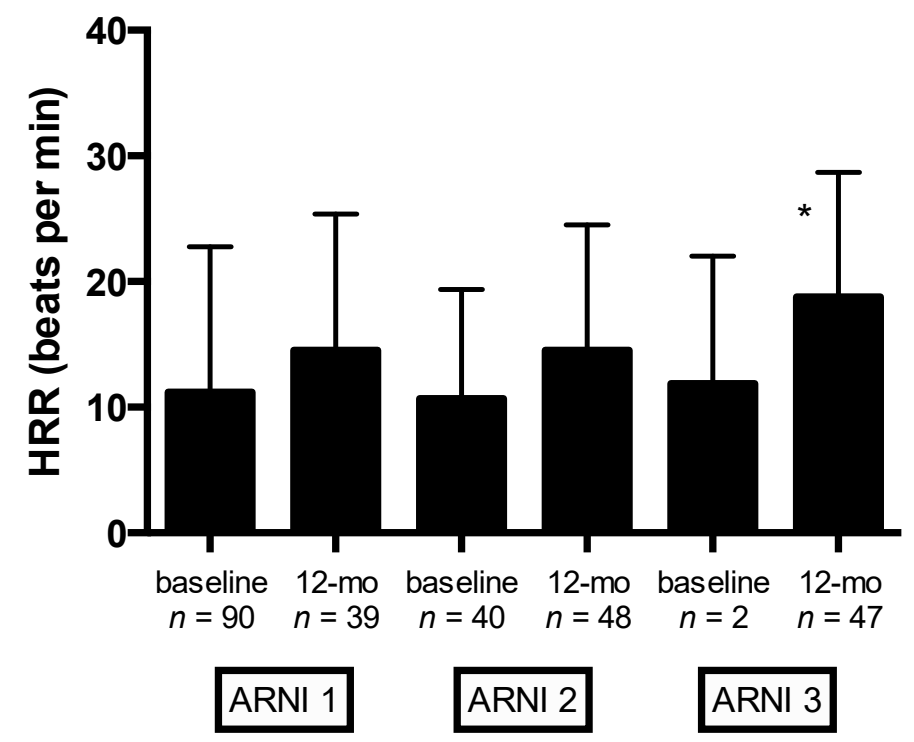

Figure 3. Baseline and 12-month follow-up values of HRR stratified by ARNI dose. Captions: angiotensin receptor neprilysin inhibitor (ARNI) dose $\left(\mathrm{ARNI}_{1}=24 / 26 \mathrm{mg}\right.$ bid, $\mathrm{ARNI}_{2}=49 / 51 \mathrm{mg}$ bid, and $\mathrm{ARNI}_{3}=97 / 103 \mathrm{mg}$ bid); HRR, heart rate recovery; ${ }^{*} p=0.009$.

No correlation between changes in HRR (bpm) and changes in systolic blood pressure (SBP) $(\mathrm{mmHg})$ was found $(r=-0.022, p=0.850)$. Changes in HRR (bpm) were significantly correlated to changes in VE/ $\mathrm{VCO}_{2 \text { slope }}(r=-0.330 ; p=0.003)$ (Figure 4). After adjusting for age, NYHA class, diabetes, atrial fibrillation, CRT/pacemaker, NT-proBNP levels, drug therapy (beta-blockers, ivabradine, 
and loop diuretics use), maximal ARNI dose reached, and changes in $\mathrm{VO}_{2 \text { peak }}$, multivariate analysis showed that changes in HRR were significantly associated to changes in $\mathrm{VE} / \mathrm{VCO}_{2 \text { slope }}(\mathrm{B}=-0.975$, $\mathrm{SE}=0.364$, Bstd $=-0.304, p=0.009$ ).

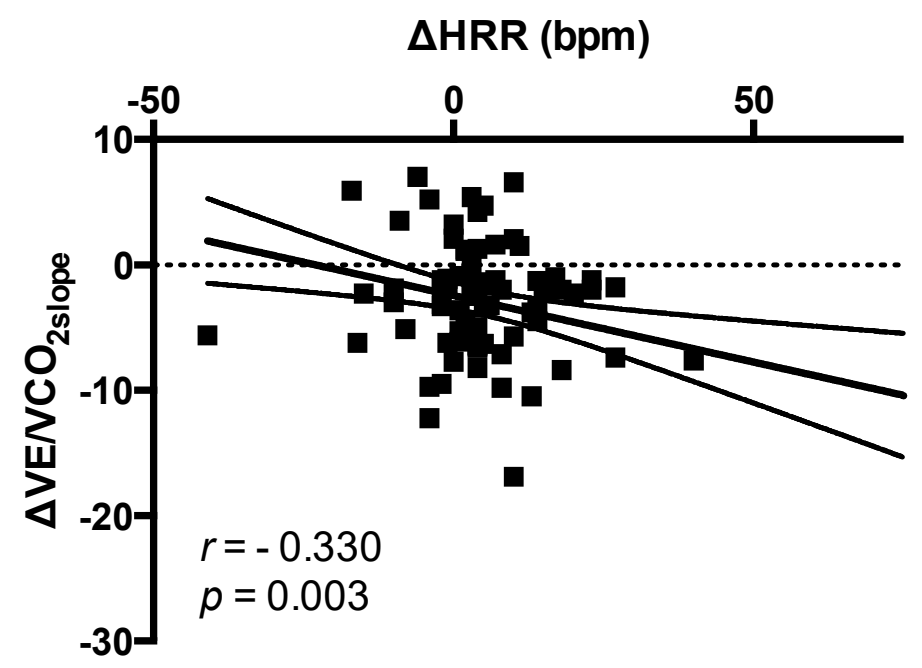

Figure 4. Correlation between changes in $\mathrm{HRR}$ and changes in $\mathrm{VE} / \mathrm{VCO}_{2 \text { slope. Captions: HRR: heart }}$ rate recovery; $\mathrm{VE} / \mathrm{VCO}_{2 \text { slope }}$ : the slope of increase in ventilation over carbon dioxide output.

Finally, at a median follow-up of $26 \pm 6.7$ months, the mortality rate was $10 \%$ (13 cardiovascular deaths), and 42 HF hospitalizations occurred. No significant association between ARNI dose or changes in HRR and these hard endpoints was found.

\section{Discussion}

This study showed a significant improvement in autonomic function as measured by HRR in HFrEF patients undergoing S/V treatment for 1 year. Autonomic function ameliorating, as expressed by the increase in HRR, was associated to the improvement in cardiopulmonary function.

To the best of our knowledge, this is the first study reporting beneficial effects on autonomic function in HFrEF patients undergoing S/V therapy. Previous studies showed that autonomic function is favorably improved by structured exercise training in patients after acute myocardial infarction or chronic heart failure [27-30]. However, exercise-induced improvement in cardiopulmonary and autonomic function is lost when exercise sessions is abandoned [28]. In a recent meta-analysis including patients with coronary artery disease, exercise-based cardiac resynchronization (CR) improved post-exercise parasympathetic function, with greater effects in younger patients with coronary artery disease and in patients who have undergone percutaneous intervention [30]. Notably, the overall effect size showed significant differences in HRR $(\mathrm{MD}=+5.35$; 95\% confidence interval $(\mathrm{CI})=4.08-6.61 \mathrm{bpm})$ in favor of the exercise-based CR group [30].

In this study, HFrEF patients on S/V therapy showed a significant increase in HRR (+6.0 beats/min, $p=0.004)$ that could likely be ascribed to the effect of $S / V$ therapy. Future studies are encouraged in order to investigate whether combined strategies (S/V therapy plus structured exercise training) might improve outcome in HFrEF patients.

HRR is a powerful predictor of all-cause mortality in patients with chronic heart failure [31-34]. The mechanisms involved in mortality reduction are probably multifactorial, and likely include a central mechanism activated by the release of inhibitory commands from the motor cortex to the parasympathetic center [35], or afferent stimulation from baroreflex or chemoreflex functions [36]. Imai et al. [37] demonstrated that a release of inhibitory central command rather than baro- or chemo-receptor stimulation may play a key role in vagal reactivation after exercise, because the heart rate response early during recovery (i.e., the first $30 \mathrm{~s}$ ) minimally depends on exercise intensity. 
Both enhanced post-infarction inflammatory response [38,39] and autonomic dysfunction [40-43] are associated with LV remodelling and poor clinical outcomes. Postinfarction healing is a highly regulated process. When post-infarction inflammatory response is efficient, a scar with tensile strength is properly formed, preventing the expansion of the infarction area, with consequent lower incidence of decompensating heart failure. Despite the key role of the healing process in post-infarction LV remodeling, the mechanisms underlying the initiation and regulation of these processes remain to be elucidated. Experimental models showed that $\mathrm{S} / \mathrm{V}$ attenuates cardiac remodeling and dysfunction after myocardial infarction by reducing cardiac fibrosis and hypertrophy [44,45]. The same mechanicistic pathways might be at the basis of the favorable effect of $\mathrm{S} / \mathrm{V}$ therapy on cardiac remodeling and inflammatory status in HFrEF patients and, consequently, responsible for the impressive outcome improvement [10].

Although this cohort of HFrEF patients did not undergo structured exercise-based cardiac rehabilitation programs, they were encouraged to maintain an adequate level of physical activity according to guidelines. It is possible that $\mathrm{S} / \mathrm{V}$ therapy by ameliorating exercise tolerance could favor the engagement of leisure time physical activity with a potential protective beneficial effect on arrhythmias and mortality. The PARADIGM-HF trial [10] showed a reduced risk for sudden death $(\mathrm{HR}=0.80,95 \% \mathrm{CI}=0.68-0.94, p=0.008)$ in HFrEF patients treated with $\mathrm{S} / \mathrm{V}$. Because only $15 \%$ of HF patients from PARADIGM-HF trial in both arms had implantable cardioverter-defibrillator (ICD), it could be hypothesized that the improvement in sudden death could be related to the improvement in autonomic function and its related protective antiarrhytmic effect. In our cohort, $76 \%$ of $\mathrm{HFrEF}$ patients had ICD and no major arrhythmic episodes were detected at device check.

EOV, a cyclic fluctuation of minute ventilation and expired gas kinetics occurring during exercise, is a marker of HF severity, and predicts either mortality or morbidity [26]. Interestingly, S/V showed a significant reduction in EOV detection at CPET (28 EOV detected at baseline CPET vs. 9 EOV detected at 12 -month follow-up, $p<0.001$ ). It could be hypothesized that the beneficial effect on autonomic function may translate to a more efficient and coordinated ventilation pattern. Future studies are eagerly awaited in order to establish whether EOV improvement might improve prognosis in HFrEF patients treated with $\mathrm{S} / \mathrm{V}$.

The relatively small sample size of patients, predominantly adult males, might limit the conclusion of the study. In addition, HRR is only an indirect, although widely accepted, reflection of sympathovagal balance. Furthermore, this study had no statistical power to analyze the relationship between HRR and prognosis. Conversely, the present study for the first time reported beneficial effects on autonomic function (as measured by HRR) in HFrEF patients undergoing S/V therapy.

\section{Conclusions}

In conclusion, 1-year S/V therapy improves autonomic function, functional capacity, and ventilatory pattern in HFrEF patients. Whether these findings might translate into beneficial effects on prognosis and outcome remains to be elucidated.

Author Contributions: Conceptualization, F.G., G.V., and C.V.; Data curation, G.V., A.D.L., F.P., G.D., F.C., S.S., M.G., G.I., and F.M.S.; Formal analysis, F.G., R.C., A.P., and A.D.; Funding acquisition, C.N. and F.M.S.; Investigation, F.C. and E.V.; Methodology, A.D.L., A.O., F.P., R.C., G.R., C.N., E.V., M.G., G.I., A.D., C.V., and F.M.S.; Resources, E.V.; Software, A.P., C.T., C.N., and S.S.; Validation, G.D.; Visualization, C.T. and G.R. Writing-original draft, F.G., E.V., and F.M.S.; Writing-review \& editing, G.V., M.P., M.G., G.I., A.D., C.V., and F.M.S. All authors have read and agreed to the published version of the manuscript.

Conflicts of Interest: The authors declare no conflict of interest. 


\section{References}

1. Peçanha, T.; Silva-Júnior, N.D.; Forjaz, C.L. Heart rate recovery: Autonomic determinants, methods of assessment and association with mortality and cardiovascular diseases. Clin. Physiol. Funct. Imag. 2014, 34, 327-339. [CrossRef] [PubMed]

2. Peçanha, T.; Bartels, R.; Brito, L.C.; Paula-Ribeiro, M.; Oliveira, R.S.; Goldberger, J.J. Methods of assessment of the post-exercise cardiac autonomic recovery: A methodological review. Int. J. Cardiol. 2017, 227, 795-802. [CrossRef] [PubMed]

3. Coote, J.H. Recovery of heart rate following intense dynamic exercise. Exp. Physiol. 2010, 95, 431-440. [CrossRef] [PubMed]

4. Goldberger, J.J.; Johnson, N.P.; Subacius, H.; Ng, J.; Greenland, P. Comparison of the physiologic and prognostic implications of the heart rate versus the RR interval. Heart Rhythm 2014, 11, 1925-1933. [CrossRef] [PubMed]

5. Kannankeril, P.J.; Le, F.K.; Kadish, A.H.; Goldberger, J.J. Parasympathetic effects on heart rate recovery after exercise. J. Investig. Med. 2004, 52, 394-401. [CrossRef]

6. Dewland, T.A.; Androne, A.S.; Lee, F.A.; Lampert, R.J.; Katz, S.D. Effect of acetylcholinesterase inhibition with pyridostig-mine on cardiac parasympathetic function in sedentary adults and trained athletes. Am. J. Physiol. Heart Circ. Physiol. 2007, 293, 86-92. [CrossRef]

7. Pepper, G.S.; Lee, R.W. Sympathetic activation in heart failure and its treatment with beta-blockade. Arch. Intern. Med. 1999, 159, 225-234. [CrossRef]

8. Rengo, G.; Galasso, G.; Femminella, G.D.A.; Parisi, V.; Zincarelli, C.; Pagano, G.; De Lucia, C.; Cannavo, A.; Liccardo, D.; Marciano, C.; et al. Reduction of lymphocyte G protein-coupled receptor kinase-2 (GRK2) after exercise training predicts survival in patients with heart failure. Eur. J. Prev. Cardiol. 2014, 21, 4-11. [CrossRef]

9. Rengo, G.; Parisi, V.; Femminella, G.D.A.; Pagano, G.; de Lucia, C.; Cannavo, A.; Liccardo, D.; Giallauria, F.; Scala, O.; Zincarelli, C.; et al. Molecular aspects of the cardioprotective effect of exercise in the elderly. Aging Clin. Exp. Res. 2013, 25, 487-497. [CrossRef]

10. McMurray, J.J.; Packer, M.; Desai, A.S.; Gong, J.; Lefkowitz, M.P.; Rizkala, A.R.; Rouleau, J.L.; Shi, V.C.; Solomon, S.D.; Swedberg, K.; et al. Angiotensin-neprilysin inhibition versus enalapril in heart failure. N. Engl. J. Med. 2014, 371, 993-1004. [CrossRef]

11. Malfatto, G.; Ravaro, S.; Caravita, S.; Baratto, C.; Sorropago, A.; Giglio, A.; Tomaselli, M.; Parati, G.; Villani, A. Improvement of functional capacity in sacubitril-valsartan treated patients assessed by cardiopulmonary exercise test. Acta Cardiol. 2019, 2, 1-5. [CrossRef] [PubMed]

12. Vitale, G.; Romano, G.; Di Franco, A.; Caccamo, G.; Nugara, C.; Ajello, L.; Storniolo, S.; Sarullo, S.; Agnese, V.; Giallauria, F.; et al. Early Effects of Sacubitril/Valsartan on Exercise Tolerance in Patients with Heart Failure with Reduced Ejection Fraction. J. Clin. Med. 2019, 8, 262. [CrossRef] [PubMed]

13. Lau, C.W.; Martens, P.; Lambeets, S.; Dupont, M.; Mullens, W. Effects of sacubitril/valsartan on functional status and exercise capacity in real-world patients. Acta Cardiol. 2019, 74, 405-412. [CrossRef] [PubMed]

14. Sgorbini, L.; Rossetti, A.; Galati, A. Sacubitril/Valsartan: Effect on Walking Test and Physical Capability. Cardiology 2017, 138 (Suppl. 1), 17-20. [CrossRef]

15. Ambrosetti, M.; Abreu, A.; Corrà, U.; Davos, C.H.; Hansen, D.; Frederix, I.; Iliou, M.C.; Pedretti, R.F.; Schmid, J.P.; Vigorito, C.; et al. Secondary prevention through comprehensive cardiovascular rehabilitation: From knowledge to implementation, 2020 update, A position paper from the Secondary Prevention and Rehabilitation Section of the European Association of Preventive Cardiology. Eur. J. Prev. Cardiol. 2020, 30, 2047487320913379. [CrossRef] [PubMed]

16. Ponikowski, P.; Voors, A.A.; Anker, S.D.; Bueno, H.; Cleland, J.G.F.; Coats, A.J.S.; Falk, V.; Gonzalez-Juanately, J.R.; Harjola, V.P.; Jankowska, E.A.; et al. ESC Scientific Document Group, 2016 ESC Guidelines for the diagnosis and treatment of acute and chronic heart failure: The Task Force for the diagnosis and treatment of acute and chronic heart failure of the European Society of Cardiology (ESC) Developed with the special contribution of the Heart Failure Association (HFA) of the ESC. Eur. Heart J. 2016, 37, 2129-2200.

17. Giallauria, F.; Cirillo, P.; Lucci, R.; Pacileo, M.; De Lorenzo, A.; D’Agostino, M.; Moschella, S.; Psaroudaki, M.; Del Forno, D.; Orio, F.; et al. Left ventricular remodelling in patients with moderate systolic dysfunction after 
myocardial infarction: Favourable effects of exercise training and predictive role of N-terminal pro-brain natriuretic peptide. Eur. J. Cardiovasc. Prev. Rehabil. 2008, 15, 113-118. [CrossRef]

18. Giallauria, F.; Lucci, R.; De Lorenzo, A.; D'Agostino, M.; Del Forno, D.; Vigorito, C. Favourable effects of exercise training on $\mathrm{N}$-terminal pro-brain natriuretic peptide plasma levels in elderly patients after acute myocardial infarction. Age Ageing 2006, 35, 601-607. [CrossRef]

19. Lang, R.M.; Bierig, M.; Devereux, R.B.; Flachskampf, F.A.; Foster, E.; Pellikka, P.A.; Picard, M.H.; Roman, M.J.; Seward, J.; Shanewise, J.; et al. Recommendations for chamber quantification. Eur. J. Echocardiogr. 2006, 7, 79-108. [CrossRef]

20. Tsang, T.S.; Abharayatna, W.P.; Barnes, M.E.; Miyasaka, Y.; Gersh, B.J.; Bailey, K.R.; Cha, S.S.; Seward, J.B. Prediction of cardiovascular outcomes with left atrial size. J. Am. Coll. Cardiol. 2006, 47, 1018-1023. [CrossRef]

21. Giallauria, F.; Galizia, G.; Lucci, R.; D’Agostino, M.; Vitelli, A.; Maresca, L.; Orio, F.; Vigorito, C. Favourable effects of exercise-based cardiac rehabilitation after acute myocardial infarction on left atrial remodeling. Int. J. Cardiol. 2009, 136, 300-306. [CrossRef] [PubMed]

22. Quinones, M.A.; Otto, C.M.; Stoddard, M.; Waggoner, A.; Zoghbi, W.A.; Doppler Quantification Task Force of the Nomenclature and Standards Committee of the American Society of Echocardiography. Recommendations for quantification of Doppler echocardiography: A report from the Doppler Quantification Task Force of the Nomenclature and Standards Committee of the American Society of Echocardiography. J. Am. Soc. Echocardiogr. 2002, 15, 167-184. [CrossRef] [PubMed]

23. Garcia, M.J.; Thomas, J.D.; Klein, A.L. New Doppler echocardiographic applications for the study of diastolic function. J. Am. Coll. Cardiol. 1998, 32, 865-875. [CrossRef]

24. Wassermann, K.; Hansen, J.E.; Sue, D.Y.; Whipp, B.J. Normal values. In Principles of Exercise Testing and Interpretation; Wassermann, K., Hansen, J.E., Sue, D.Y., Whipp, B.J., Eds.; Lea \& Febiger: Philadelphia, PA, USA, 2004; Volume 1987, pp. 72-85.

25. Beaver, W.L.; Wasserman, K.; Whipps, B.J. A new method for detecting anaerobic threshold by gas exchange. J. Appl. Physiol. 1986, 60, 2020-2027. [CrossRef]

26. Corrà, U.; Giordano, A.; Bosimini, E.; Mezzani, A.; Piepoli, M.; Coats, A.J.; Giannuzzi, P. Oscillatory ventilation during exercise in patients with chronic heart failure: Clinical correlates and prognostic implications. Chest 2002, 121, 1572-1580. [CrossRef]

27. Giallauria, F.; Lucci, R.; Pietrosante, M.; De Lorenzo, A.; Gargiulo, G.; D’Agostino, M.; Gerundo, G.; Abete, P.; Rengo, F.; Vigorito, C. Exercise-based Cardiac Rehabilitation improves Heart Rate Recovery in Elderly Patients after Acute Myocardial Infarction. J. Gerontol. Ser. A Biol. Sci. Med. Sci. 2006, 61, 713-717. [CrossRef]

28. Giallauria, F.; De Lorenzo, A.; Pilerci, F.; Manakos, A.; Lucci, R.; Psaroudaki, M.; D’Agostino, M.; Del Forno, D.; Vigorito, C. Long-Term Effects of Cardiac Rehabilitation On End-Exercise Heart Rate Recovery After Myocardial Infarction. Eur. J. Cardiovasc. Prev. Rehabil. 2006, 13, 544-550. [CrossRef]

29. Pearson, M.J.; Smart, N.A. Exercise therapy and autonomic function in heart failure patients: A systematic review and meta-analysis. Heart Fail. Rev. 2018, 23, 91-108. [CrossRef]

30. Manresa-Rocamora, A.; Ribeiro, F.; Sarabia, J.M.; Íbias, J.; Oliveira, N.L.; Vera-Garcia, F.J.; Moya-Ramón, M. Exercise-based cardiac rehabilitation and parasympathetic function in patients with coronary artery disease: A systematic review and meta-analysis. Clin. Anton. Res. 2020. [CrossRef]

31. Tang, Y.D.; Dewland, T.A.; Wencker, D.; Katz, S.D. Post-exercise heart rate recovery independently predicts mortality risk in patients with chronic heart failure. J. Card. Fail. 2009, 15, 850-855. [CrossRef]

32. Sheppard, R.J.; Racine, N.; Roof, A.; Ducharme, A.; Blanchet, M.; White, M. Heart rate recovery-A potential marker of clinical outcomes in heart failure patients receiving beta-blocker therapy. Can. J. Cardiol. 2007, 23, 1135-1138. [CrossRef]

33. Cahalin, L.P.; Forman, D.E.; Chase, P.; Guazzi, M.; Myers, J.; Bensimhon, D.; Peberdy, M.A.; Ashley, E.; West, E.; Arena, R. The prognostic significance of heart rate recovery is not dependent upon maximal effort in patients with heart failure. Int. J. Cardiol. 2013, 168, 1496-1501. [CrossRef] [PubMed]

34. Hajdusek, P.; Kotrc, M.; Kautzner, J.; Melenovsky, V.; Benesova, E.; Jarolim, P.; Benes, J. Heart rate response to exercise in heart failure patients: The prognostic role of metabolic-chronotropic relation and heart rate recovery. Int. J. Cardiol. 2017, 228, 588-593. [CrossRef] [PubMed]

35. Toska, K.; Eriksen, M. Peripheral vasoconstriction shortly after onset of moderate exercise in human. J. Appl. Physiol. 1994, 77, 1519-1525. [CrossRef] 
36. Walgenbach, S.C.; Donald, D.E. Inhibition by carotid baroreflex of exercise-induced increases in arterial pressure. Circ. Res. 1983, 52, 253-262. [CrossRef]

37. Imai, K.; Sato, H.; Hori, M.; Kusuoka, H.; Ozaki, H.; Yokoyama, H. Vagally mediated heart rate recovery after exercise is accelerated in athletes but blunted in patients with chronic heart failure. J. Am. Coll. Cardiol. 1994, 24, 1529-1535. [CrossRef]

38. Giallauria, F.; Cirillo, P.; Lucci, R.; Pacileo, M.; D’Agostino, M.; Maietta, P.; Vitelli, A.; Chiariello, M.; Vigorito, C. Autonomic Dysfunction is associated with High Mobility Group Box-1 Levels in Patients after Acute Myocardial Infarction. Atherosclerosis 2010, 208, 280-284. [CrossRef]

39. Frangogiannis, N.G.; Smith, C.W.; Entman, M.L. The inflammatory response in myocardial infarction. Cardiovasc. Res. 2002, 53, 31-47.

40. Cole, C.R.; Blackstone, E.H.; Pashkow, F.J.; Snader, C.E.; Lauer, M.S. Heart-rate recovery immediately after exercise as a predictor of mortality. N. Engl. J. Med. 1999, 341, 1351-1357. [CrossRef]

41. Nishime, E.O.; Cole, C.R.; Blackstone, E.H.; Pashkow, F.J.; Lauer, M.S. Heart-rate recovery and treadmill exercise score as a predictors of mortality in patients referred for exercise ECG. JAMA 2000, 284, 1392-1398. [CrossRef]

42. Watanabe, J.; Thamilarasan, M.; Blackstone, E.H.; Thomas, J.D.; Lauer, M.S. Heart rate recovery immediately after treadmill exercise and left ventricular systolic dysfunction as predictors of mortality. Circulation 2001, 104, 1911-1916. [CrossRef] [PubMed]

43. Vivekananthan, D.P.; Blackstone, E.H.; Pothier, C.E.; Lauer, M.S. Heart rate recovery after exercise is a predictor of mortality, independent of the angiographic severity of coronary disease. J. Am. Coll. Cardiol. 2003, 42, 831-838. [CrossRef]

44. von Lueder, T.G.; Wang, B.H.; Kompa, A.R.; Huang, L.; Webb, R.; Jordaan, P.; Atar, D.; Krum, H. Angiotensin receptor neprilysin inhibitor LCZ696 attenuates cardiac remodeling and dysfunction after myocardial infarction by reducing cardiac fibrosis and hypertrophy. Circ. Heart Fail. 2015, 8, 71-78. [CrossRef] [PubMed]

45. Suematsu, Y.; Jing, W.; Nunes, A.; Kashyap, M.L.; Khazaeli, M.; Vaziri, N.D.; Moradi, H. LCZ696 (Sacubitril/Valsartan), an Angiotensin-Receptor Neprilysin Inhibitor, Attenuates Cardiac Hypertrophy, Fibrosis, and Vasculopathy in a Rat Model of Chronic Kidney Disease. J. Card. Fail. 2018, 24, $266-275$. [CrossRef] [PubMed]

(C) 2020 by the authors. Licensee MDPI, Basel, Switzerland. This article is an open access article distributed under the terms and conditions of the Creative Commons Attribution (CC BY) license (http://creativecommons.org/licenses/by/4.0/). 